Ann. Biol. anim. Bioch. Biophys., I973, 13 (4), 553-563.

\title{
ÉVOLUTION DE LA COMPOSITION AZOTÉE DES MUSCLES PSOAS MAJOR ET LONGISSIMUS DORSI, DE LA NAISSANCE A 3 SEMAINES CHEZ LE LAPIN
}

\author{
P. VIGNERON \\ avec la collaboration technique de L. MARger \\ Station de Physiologie animale, I. N.R. A., \\ École nationale supérieure agronomique, \\ Place Viala \\ 34060 Montpellier Cedex
}

\section{RÉSUMÉ}

L'évolution des proportions des principales fractions azotées musculaires a été étudiée de la naissance à 22 jours pour le muscle psoas major d'une souche de lapins communs d'une part, et pour le psoas major et le longissimus dorsi d'une souche de Néo-Zélandais d'autre part. Pendant cette période, on note, par rapport à l'azote total, une augmentation importante de l'azote myofibrillaire, les autres fractions présentant une stagnation ou même une diminution relative. Dès le I I $^{\mathrm{e}}$ jour, la composition azotée relative du muscle adulte semble atteinte. On retrouve ce type d'évolution quel que soit le muscle étudié. dorsi.

Cependant, le psoas major semble avoir un développement plus précoce que le longissimus

Au stade de I I jours, une différence de composition, significative au seuil 5 p. Ioo, n'a pu être mise en évidence entre mâles et femelles que pour l'azote sarcoplasmique dans le psoas major des Néo-Zélandais. L'origine de cette différence est encore inconnue.

\section{INTRODUCTION}

La composition biochimique relative du muscle squelettique peut présenter des variations tenant à l'espèce, la race, l'état physiologique de l'individu (âge, sexe entraînement physique,...) et la nature du muscle étudié (rouge, blanc, mixte). Dans des conditions courantes d'élevage, les proportions relatives des constituants azotés du muscle semblent déjà fixées dès la $3^{\mathrm{e}}$ semaine chez le Lapin, comme nous l'avons constaté lors d'un précédent travail (BARON, VIGNERON, I970). Nous avions également relevé une différence significative entre les composition azotée du psoas 
major des mâles et des femelles. Il était intéressant d'observer l'évolution des fractions azotées musculaires au cours de la période où le jeune animal commence à se déplacer, de la naissance à la $3^{\mathbf{e}}$ semaine postnatale. Nous l'avons étudiée pour un muscle de type blanc (psoas major) et un muscle mixte (longissimus dorsi) ; nous avons également recherché l'existence éventuelle d'une différence liée au sexe, perceptible dès cette période.

\section{MATÉRIEL ET MÉTHODES}

\section{A. - Matériel animal}

La composition du psoas majcr a été étudiée chez une souche de lapins "communs ", sélectionnée au laboratoire. Dans un travail antérieur (BARON, VIGNERON, 1970), nous avons déjà décrit l'évolution de la composition du psoas major, après l'âge de 3 semaines, chez des animaux appartenant à cette même souche.

Comparativement, nous avons étudié la composition du psoas major et du longissimus dorsi d'une souche de lapins Néo-Zélandais élevés dans les mêmes conditions d'environnement, décrites précédemment par PRUD'Hon (1967).

Les lapins "communs" et Néo-Zélandais ont un format adulte très voisin (4 kg environ).

Les stades retenus pour l'étude, ainsi que le nombre et le sexe des individus abattus à chaque stade sont rassemblés dans le tableau I.

\section{TABLEAU I}

Effectif, sexe, et race des animaux utilisés

\begin{tabular}{|c|c|c|c|c|c|c|}
\hline Stades & Naissance & 5 jours & \multicolumn{2}{|c|}{11 jours } & 16 jours & 22 jours \\
\hline $\begin{array}{l}\text { Psoas major } \\
\text { Lapins communs }\end{array}$ & 5 웅 & 4 우 & \multicolumn{2}{|c|}{5 ㅇ } & 49 & 49 \\
\hline $\begin{array}{c}\text { Psoas major } \\
\text { Lapins Néo-Zelandais }\end{array}$ & $5 q-20$ & 5 ㅇ & 60 & 11 우 & $4 q-2 \delta$ & 3 우 -2 万 \\
\hline $\begin{array}{l}\text { Longissimus dorsi } \\
\text { Lapins Néo-Zélandais }\end{array}$ & 4 우 $-2 \delta$ & 5 우 & $6 \delta^{\circ}$ & 10 ㅇ & $49-2 \delta$ & 3 우 $-2 \sigma^{\star}$ \\
\hline
\end{tabular}

Nous n'avons analysé séparément la composition des muscles des individus mâles et femelles que chez les lapins Néo-Zélandais âgés de r I jours, ce stade étant retenu comme suffisamment éloigné de la naissance et du sevrage pour limiter les variations individuelles qui leur sont parfois associées.

L'étude du longissimus dorsi a été réalisée compte tenu de la nature différente de ses fibres : le psoas major du Lapin dont la croissance a été étudiée par Meara (1947), est un muscle qualifié d'entièrement blanc par ARANGio et HaGSTROM (1969). Nous n'y avons pour notre part décelé que de rares fibres rouges au cours d'une étude histochimique. Le longissimus dorsi, par contre, est un muscle mixte comportant une quantité importante de fibres rouges.

Des études effectuées dans notre station montrent que la croissance pondérale de ces deux muscles rachidiens, situés de part et d'autre de l'axe vertébral, présente de nettes analogies. Relativement à celle de la masse musculaire squelettique totale, leur croissance s'effectue en deux phases pour lesquelles les coefficients d'allométrie calculés, rappelés dans le tableau ci-après, sont très voisins. (CANTIER et al, 1973). On peut cependant observer un changement d'allométrie plus précoce pour le longissimus dorsi. 


\section{TABLEAU 2}

Coefficients des droites d'allométrie de croissance calculés pour le longissimus dorsi et le psoas major

(Variable de référence : poids du système musculaire total)

\begin{tabular}{c|c|c|c|c|}
\hline & $\begin{array}{c}\text { Coefficient } \\
\text { d'allométrie } \\
\text { moyen }\end{array}$ & $\begin{array}{c}\text { Coefficients d'allométrie } \\
\text { réels }\end{array}$ & $\begin{array}{c}\text { Intervalle de changement } \\
\text { d'allométrie } \\
\text { (poids total de l'ensemble } \\
\text { des muscles) }\end{array}$ \\
\hline Longissimus dorsi & $1,24 \pm 0,04$ & $1,30 \pm 0,07$ & $0,82 \pm 0,14$ & $735-760 \mathrm{~g}$ \\
\hline Psoas major & $1,18 \pm 0,04$ & $1,20 \pm 0,07$ & $0,82 \pm 0,43$ & $988-1108 \mathrm{~g}$ \\
\hline \hline
\end{tabular}

\section{B. - Méthodes d'analyse}

Elles ont déjà été exposées dans un précédent article (BARON, VignERoN, I97o). Elles reposent sur une solubilisation différentielle de fractions azotées, à l'aide de milieux de forces ioniques différentes (HELANDER, I957). L'utilisation d'un broyeur homogénéiseur Ultra-Turrax a permis une meilleure extraction des diverses fractions que la simple macération de coupes de tissu. La répétabilité des résultats obtenus ainsi s'est révélée satisfaisante.

Outre l'azote total, cette méthode permet d'estimer 4 fractions :

- azote myofibrillaire ( $\mathrm{N}$ myo) ;

- azote sarcoplasmique ( $\mathrm{N}$ sarco) ;

- azote du stroma (N stroma) ;

- azote non protéique (N NP).

\section{RÉSULTATS}

Sur les tableaux 3,4 et 5 sont indiqués le poids vif et le poids vif vide moyens pour chaque stade. Pour le Lapin commun (tabl. 3) on remarque un écart-type important aux stades 16 et 22 jours, en rapport avec un nombre peu élevé d'individus, formant des lots peu homogènes. C'est le cas en particulier au stade 22 jours où le poids vif et le poids vif vide d'un des animaux était de $40 \mathrm{p}$. Ioo inférieur à la moyenne des autres.

De plus, nous avons fait figurer dans les tableaux 3, 4 et 5, pour chaque stade, les valeurs des diverses fractions azotées, exprimées en concentration dans le tissu frais, et, pour éliminer le facteur de variation important introduit par l'évolution rapide de la teneur en eau de l'organisme et les tissus qui le constituent, pendant la période étudiée (VIGNERON et al., I97I), nous avons également porté les fractions azotées en $p$. roo de l'azote total. Enfin le rapport $N$ myofibrillaire/ $N$ sarcoplasmique représente le rapport de la masse des protéines contractiles sur celle des protéines sarcoplasmiques, qui sont en majeure partie des enzymes (ScOPES, I970).

L'évolution des concentrations moyennes des différentes fractions azotées a été représentée en fonction du poids vif vide en coordonnées arithmétiques (fig. I). 

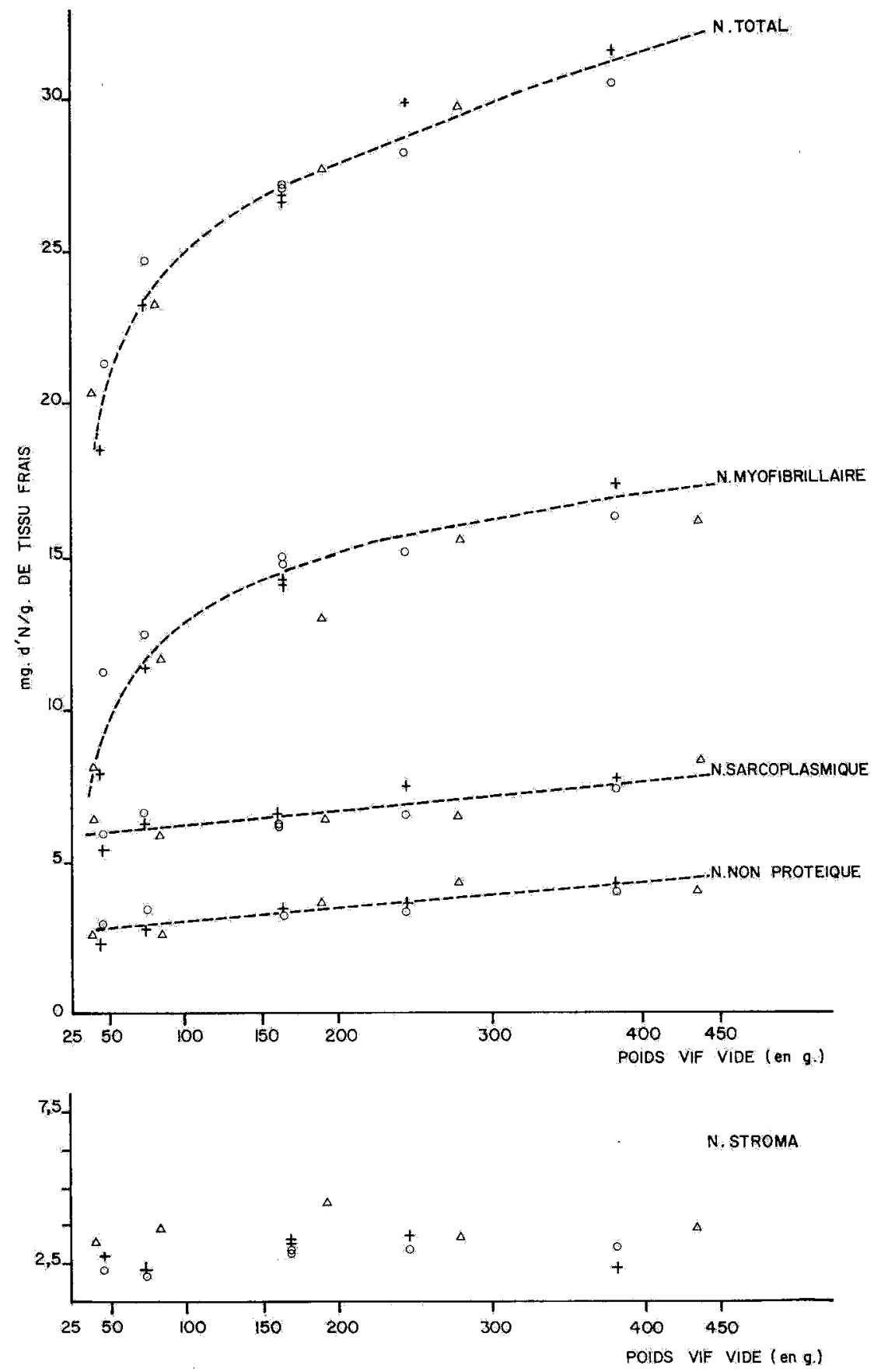

Fig. I 


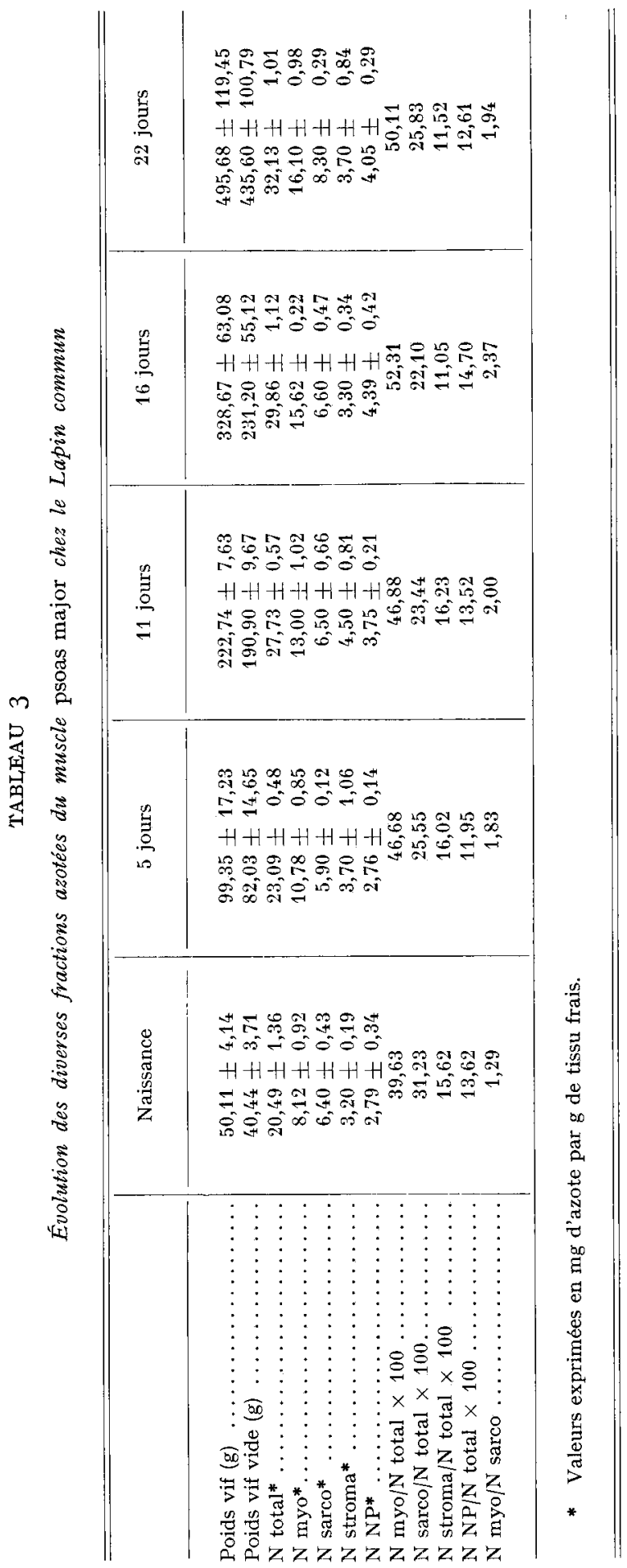




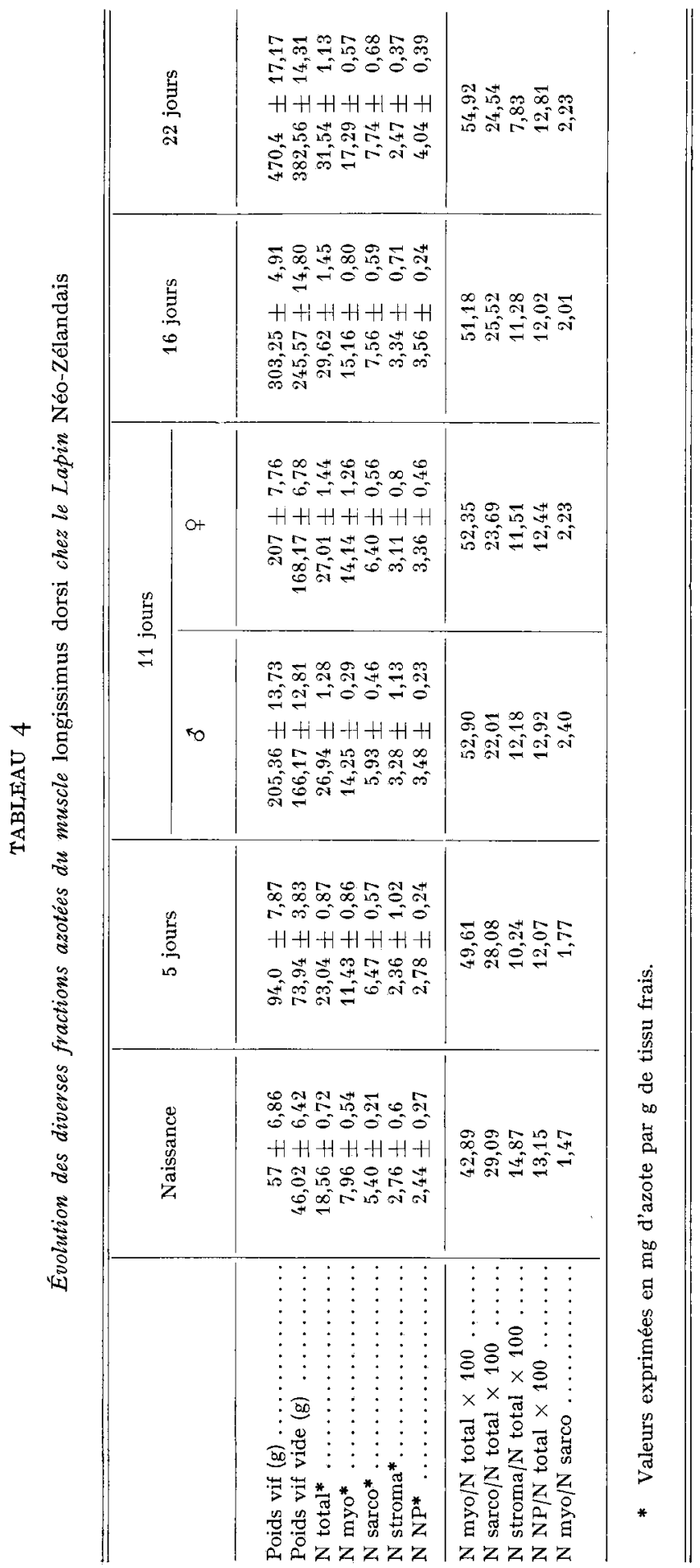




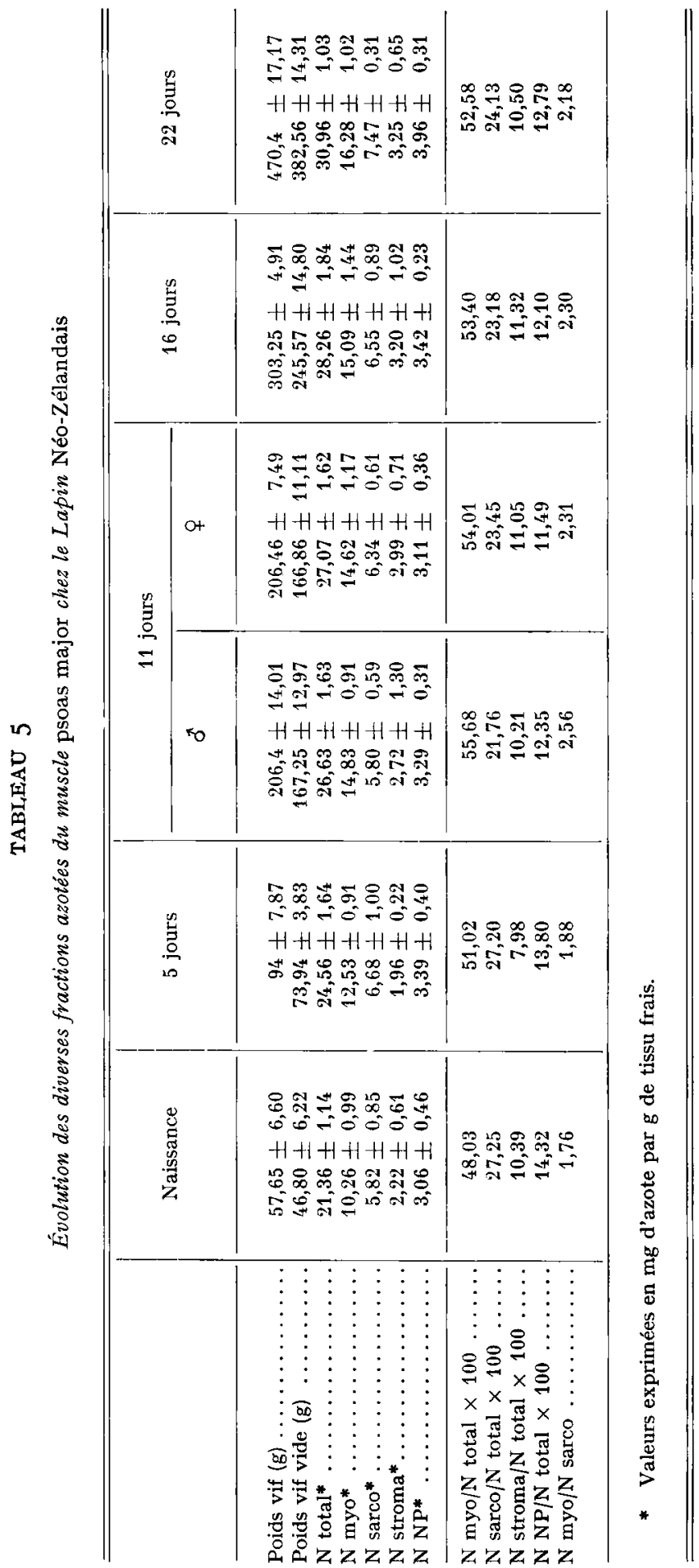


Pour le psoas major du Lapin commun (tabl. 3), on note dans le tissu frais une évolution plus importante pour les concentrations en azote total et en azote myofibrillaire, qui passent respectivement de 20,5 à $32 \mathrm{mg} / \mathrm{g}$, et de 8 à $\mathrm{I} 6 \mathrm{mg} / \mathrm{g}$ de tissu frais. Les concentrations en azote sarcoplasmique et en azote non protéique montrent une augmentation légère, alors que la concentration en azote du stroma ne varie pas de façon significative.

Les valeurs de ces fractions azotées, exprimées en p. roo de l'azote total, montrent une nette augmentation de la part relative de l'azote myofibrillaire, qui passe de 40 à plus de $50 \mathrm{p}$. Ioo. Cela entraîne une diminution des proportions de l'azote sarcoplasmique (de 3I à 25 p. Ioo) et de l'azote du stroma (de I5 à II p. IOo). L'azote non protéique représente un pourcentage peu variable de l'azote total, de l'ordre de I3 p. Ioo.

Chez le Néo-Zélandais, les valeurs des différentes fractions, relevées pour le longissimus dorsi, ainsi que leur évolution (tabl. 4) sont voisines de celles obtenues pour le psoas major, chez le Lapin commun. Par contre, pour le muscle psoas major de cette race (tabl. 5), les évolutions observées sont moins importantes du fait des valeurs plus élevées des concentrations en azote myofibrillaire aux 3 premiers stades (naissance, 5 jours, II jours).

Au I I $^{\mathrm{e}}$ jour, chez les Néo-Zélandais, on n'observe que de faibles différences entre les diverses valeurs des fractions azotées du psoas major et du longissimus dorsi. Comme nous l'avons noté chez des animaux plus âgés, les mâles présentent des teneurs légèrement supérieures pour l'azote myofibrillaire, inférieures pour l'azote sarcoplasmique. Mais ces différences ne sont significatives que pour l'azote sarcoplasmique du psoas major (différence significative au seuil 5 p. Ioo).

Nous avons représenté (fig. I) l'évolution des concentrations de l'azote total et ses diverses fractions pour l'ensemble des muscles étudiés. Le poids vif vide a été choisi comme variable de référence, de préférence au temps, du fait des différences

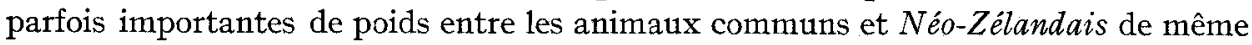
âge. De plus, il présente sur le poids vif l'avantage d'être indépendant des grandes différences constatées entre individus dans l'état de réplétion de l'estomac.

On constate que l'évolution des concentrations en azote total et en azote myofibrillaire est beaucoup plus rapide pendant les II premiers jours après la naissance que par la suite. Les concentrations en azote sarcoplasmique et en azote non protéique montrent une évolution uniforme, qui s'ajuste selon une droite $y=a+b x$ où $x$ est le poids vif vide et $y$ la concentration en azote de la fraction considérée. Pour l'azote sarcoplasmique, $y=5,6 \mathrm{I}+0,005 x$ avec $b=0,005 \pm 0,0009$, et pour l'azote non protéique $y=2,7 \mathrm{I}+0,0036 x$ avec $b=0,0036 \pm 0$, 000 9. Les concentrations en azote du stroma, plus dispersées, ne semblent pas présenter de variation notable pendant la période considérée.

\section{DISCUSSION}

Alors qu'au cours de la période 3-26 semaines, nous avions enregistré peu de variations de la composition azotée du muscle psoas major (BARON, VIGNERON, I970), nous pouvons constater ici une rapide évolution postnatale des concentrations des diverses fractions azotées et de leurs proportions. 
La fraction des protéines myofibrillaires est la seule à présenter une augmentation relative après la naissance. Les autres fractions ( $N$ sarcoplasmique, $N$ du stroma) présentent au contraire une diminution, ou une très faible variation ( $\mathrm{N}$ non protéique) (tableaux $3,4,5$ ). Ces résultats sont d'ailleurs comparables à ceux obtenus chez le Lapin par PERRY et HARTSHORNE (I962) et par IVANov (I967) à l'aide de techniques différentes.

Les proportions des fractions azotées semblent ainsi atteindre dès le II ${ }^{\mathrm{e}}$ jour des valeurs très voisines de ce qu'elles sont chez l'adulte. Ces résultats sont à rapprocher de ceux obtenus par RoBInson (I952) qui montre chez le Poussin une augmentation rapide des proportions d'azote myofibrillaire après l'éclosion. Chez le Poussin également, Dickerson (I960), Mizuno et al. (I966) observent que les diverses fractions azotées atteignent des pourcentages proches de ceux obtenus chez l'adulte, dès le premier mois après l'éclosion. Herrmann et Nichotas (I948), Gordon et al. (I966) chez le Rat, Dickerson et Widdowson (I960) chez le Porc, Heidander (I957) chez les bovins, mettent tous en évidence une évolution postnatale rapide des proportions des fractions myofibrillaires et sarcoplasmiques.

OGaWa (I96I) met en évidence chez le Poulet la présence d'actine dès 72 heures, et de myosine dès 96 heures après la mise en incubation. BARIL, et HERMANN (I967) trouvent une augmentation de Io fois de la quantité de myosine pendant le développement du Poussin entre le $9^{\mathrm{e}}$ jour après le début de l'incubation et le $8^{\mathrm{e}}$ jour après l'éclosion. La fusion des myoblastes, qui survient dès le $6^{\mathrm{e}}$ jour après la mise en incubation, et conduit à la formation des fibres musculaires, est accompagnée d'une synthèse active de protéines myofibrillaires (STOCKDALE et HolTZER, I96I).

Le nombre de fibres d'un muscle semble généralement fixé dès la naissance. C'est le cas chez le Lapin pour certains muscles; pour d'autres, ce nombre augmente encore légèrement au cours du premier mois de vie postnatale. (Nouguìs, I972). Il est donc probable que très vite après leur formation, les fibres puissent prendre une composition voisine de celle qu'elles auront par la suite, dans la mesure où elles participent rapidement à une activité motrice. L'influence de ce facteur activité est bien mis en évidence par le travail de DickERson et WIDDowson (Ig6o) : chez l'enfant de 5 à 7 mois, encore peu actif, les proportions des diverses fractions azotées sont encore assez nettement différentes de ce qu'elles sont chez l'adulte. D'autre part, chez des animatux ayant achevé leur croissance, l'entraînement ou l'immobilisation peuvent modifier le taux de synthèse des protéines myofibrillaires (HELANDER, I96I).

Le psoas major semble donc avoir un développement légèrement plus précoce que le longissimus dorsi puisqu'il présente à la naissance une quantité relative plus importante de protéines myofibrillaires (tab1. 4 et 5).

Cette différence s'estompe très vite par la suite et se trouve presque abolie dès le II $^{\mathrm{e}}$ jour, comme le montre bien 1'évolution du rapport $\mathrm{N}$ myofibrillaire/ $\mathrm{N}$ sarcoplasmique. Cependant, d'un point de vue qualitatif, le psoas major continue à subir des changements importants dans la nature de son équipement enzymatique (BACou, I972). Les différences observées à II jours entre mâles et femelles, sont très faibles, mais vont cependant dans le même sens que celles que nous avons relevées pour le psoas major d'animaux plus âgés. Nous ignorons encore si la plus grande quantité d'azote myofibrillaire et la plus faible quantité d'azote sarcoplasmique constatées chez les mâles sont en rapport avec une synthèse stimulée par le niveau relatif des hormones circulantes ou par l'activité physique. 
Il n'est pas possible, compte tenu du faible effectif animal, de relever des différences entre races dans l'évolution des diverses fractions azotées.

Ce travail prolonge et confirme ce que nous avions déjà mis partiellement en évidence : après la naissance, la croissance biochimique du muscle ne présente de modifications importantes dans les proportions des diverses catégories de protéines que pendant les Io premiers jours. Les changements observés par la suite sont beaucoup plus progressifs et ne jouent que sur de faibles pourcentages de l'azote total.

Reçu pour publication en mai 1973.

\section{SUMMARY}

\section{EVOLUTION OF NITROGEN COMPOSITION OF PSOAS MAJOR AND LONGISSIMUS DORSI MUSCLES IN RABBIT FROM BIRTH TO 3 WEEKS}

Evolution of proportions of the main nitrogenous muscle fractions is studied from birth to 22 days in the psoas major muscle of a common rabbit strain, and in the psoas major and longissimus dorsi of a New Zealand strain. During this period, there is large increase of myofibrillar nitrogen in relation to total nitrogen; other fractions present stagnation or even a relative decrease At day I , relative nitrogen composition of adult muscle seems to be reached. This type of evolution is found in any muscle studied.

However, the psoas major appears to develop earlier than the longissimus dorsi.

At day I I stage, there is only a difference in composition (significant at a threshold of $5 \mathrm{p}$. 100) between males and females for sarcoplasm nitrogen in the psoas major of New Zealand rabbits. The origin of this difference is unknown.

\section{RÉFÉRE,NCES BIBLIOGRAPHIQUES}

Arangio G. A., Hagstrom J. W. C., I969. The histochemical classification of rabbit hindlimb striated muscle. J. Histochem. Cytochem., 17, I27-128.

BAcou F., I972. Évolution quantitative de l'aldolase, de l'aspartate aminotransférase, de la succinate déhydrogénase et de l'acétylcholinestérase dans les muscles blancs et rouges du Lapin au cours de la période postnatale. C. R. Soc. Biol. 166, ro37-1042.

Baril F. F., HeRRMANN H., 1967. Studies of muscle development. II. Immunological and enzymatic properties and accumulation of chromatographically homogeneous myosin of the leg musculature of the developing chick. Devl. Biol., 15, 318-333.

Baron R., Vigneron P., r970. Hétérogonie biochimique des composants azotés du psoas major au cours de la croissance postanatale du Lapin. Ann. Biol. anim. Bioch. Biophys., 10, 223-238.

BRISkey E. J., CASSENS R. G., MARSh B. B., I970. The physiology and biochemistry of muscle as a food., 2, $843 \mathrm{pp}$. Wisconsin press Madison.

Cantier J., Dulor J.-P., Rouvier R., Vézinhet A., 1973. Allométrie de croissance chez le Lapin. IV. Principaux muscles de la carcasse. Ann. Biol. anim. Bioch. Biophys., (sous presse).

Dickerson J. W. T., rg6o. The effect of growth on the composition of avian muscle. Biochem. J., 75, 33-37.

Dickerson J. W. T., Widdowson E. M., I960. Chemical changes in skeletal muscle during development. Biochem. J., 74, 247-257.

Gordon E. E., KowAlski K., FRITTS M., I966. Muscle proteins and DNA in rat quadriceps during growth. Am. J.Physiol, 210, 1033-1039.

Helander E., 1957. On quantitative muscle protein determination. Acta. physiol. scand., Suppl., 141, 9-95.

HeLander E., I96I. Influence of exercise and restricted activity on the protein composition of skeletal muscle. Biochem. $J ., 78,478-482$.

HermanN H., Nicolas J. S., I948. Quantitative changes in muscle protein fractions during rat development. J.exp. Zool., 107, r65-I76. 
Ivanov I. I., I967. Organic substances of the striated muscle. Symp. Biol. Hung., 8, 89-124.

Meara P. J., 1947. Postnatal growth and development of muscle, as exemplified by the gastrocnemius and psoas muscles of the rabbit. Onderstepoort. J. vet. Sci. anim. indust., 21, 329-466.

Mizuno T., Hikami Y., Sumita $K$, rg66. The accumulation of nucleic acid and protein in the skeletal muscle of chicks durmg embryonic and post-embryonic development. Jap. J. zootech. Sci., 37, $471-477$.

Nouguès J., r972. Étude de l'évolution du nombre des fibres musculaires au cours de la croissance postnatale du muscle chez le Lapin. C. R. Soc. Biol., 166, I65-172.

OGAwA Y., 1962. Synthesis of skeletal muscle proteins in early embryos and regenerating tissue of chick and triturus. Expl. Cell. Res., 26, 269-274.

Perry S. V., Hartshorne D. J., ig63. in : Gutman E. et Hnik P. Effect of use and disuse on neuromuscular functions. Czechoslovak. Acad. Sci. Prague. 539. Cité par Briskey, I97o.

PruD'hon M., 1967. L'appétit du Lapin alimenté à sec. Bull. tech. Inf. Ingrs. Servs. agric., 219, 383-398.

Robinson D. S., 1952. Changes in the protein composition of chick muscle during development. Biochem. J., 52, 62 1-628.

Scopes E. K., 197o. in : Briskey E. J., Cassens R. G., Marsh B. B., The physiology and biochemistry of muscle as a food, 2, 47I-492. Wisconsin press. Madison.

Stockdale F. E., Holtzer H., 1961. DNA synthesis and myogenesis. Expl. Cell. Res., 21, 508-520.

Vigneron P., Baron R., Dauzier L., I97I. Évolution postnatale de la quantité d'eau et de lipides du corps et du psoas major chez le Lapin. Ann. Biol. anim. Bioch. Biophys., 11. 669-679. 